\title{
Adaptation of the Fear of COVID-19 Scale: Its Association with Psychological Distress and Life Satisfaction in Turkey
}

\author{
Begum Satici $^{1} \cdot$ Emine Gocet-Tekin $^{2} \cdot$ M. Engin Deniz ${ }^{3} \cdot$ Seydi Ahmet Satici ${ }^{1}$
}

Published online: 8 May 2020

C) Springer Science+Business Media, LLC, part of Springer Nature 2020

\begin{abstract}
The world is currently experiencing a pandemic of an infectious disease called COVID19 which has drawn global intensive attention. While global attention is largely focusing on the effects of the coronavirus on physical health, the impacts of the coronavirus on psychological health cannot be overlooked. Therefore, this study aims to adapt the Fear of COVID-19 Scale into Turkish and investigate the relationships between fear of COVID19, psychological distress, and life satisfaction. Data were collected by convenience sampling method, which allowed us to reach total 1304 participants, aged between 18 and 64 years, from 75 cities in Turkey. In the adaptation process of the Fear of COVID-19 Scale, confirmatory factor analysis, Item Response Theory, convergent validity, and reliability (Cronbach's $\alpha$, McDonald's $\omega$, Guttmann's $\lambda 6$, and composite reliability) analyses were performed. Additionally, the mediating role of psychological distress on the relationship between fear of COVID-19 and life satisfaction was tested. The unidimensionality of the 7-item scale was confirmed on a Turkish sample. Item Response Theory revealed that all items were coherent and fit with the model. The results indicated that the Turkish version of the scale had satisfactory reliability coefficients. The fear of COVID-19 was found to be associated with psychological distress and life satisfaction. Results indicated that the Turkish version of the Fear of COVID-19 Scale had strong psychometric properties. This scale will allow mental health professionals to do research on the psychological impacts of COVID-19 in Turkey.
\end{abstract}

Keywords COVID-19 $\cdot$ Fear $\cdot$ Psychological distress $\cdot$ Life satisfaction $\cdot$ Scale

Begum Satici

begum@artvin.edu.tr

1 Department of Educational Sciences, Artvin Coruh University, Artvin, Turkey

2 Department of Foreign Languages, Sakarya University, Sakarya, Turkey

3 Department of Educational Sciences, Yildiz Technical University, Istanbul, Turkey 
The novel coronavirus disease 2019 (COVID-19) epidemic first emerged in the city of Wuhan of Hubei Province, China, on December 12, 2019. Within 3 months of the initial outbreak, the virus has spread rapidly to neighboring countries and beyond, which led the World Health Organization (WHO) to declare the outbreak a pandemic on March 11, 2020 when the first coronavirus case was confirmed in Turkey. Since then, Turkey, like all countries dealing with this virus, has taken necessary measures to stop the spread of the coronavirus, including shutting down schools and closing places where people gather such as bars, shopping malls, movie theaters, gymnasiums, and other sports venues. The government in Turkey has asked its citizens to keep themselves in voluntary quarantine and stay at home to reduce the number of infections and protect our elderly and those with chronic diseases. Both the nature of the COVID-19 being highly infectious and relatively deadly and sudden unprecedented measures taken by the governments pose risks to the mental health of the individuals not only in Turkey but also in almost all countries around the world.

Scientists around the world have focused on the diagnostic and therapeutic aspects of treating COVID-19. On the other hand, there are a limited number of studies regarding the psychological impacts of COVID-19 on mental health (Mamun and Griffiths 2020; Pakpour and Griffiths 2020; Schimmenti et al. 2020; Wang et al. 2020). The outbreak of COVID-19 and its pandemic nature has caused widespread concern, fear, and anxiety (Ahorsu et al. 2020). Additionally, COVID-19 triggers fear among individuals which makes the understanding of the impact of the crisis on people's mental health crucial (Xiang 2020).

Fear and panic about COVID-19 can lead to experiences of stigmatization and social exclusion of confirmed patients, survivors, their families, and others associated with the disease which can cause an increased risk of developing mental health problems like adjustment disorder and depression (Zhang et al. 2020). Moreover, uninfected people reported that they are afraid of contacting with COVID-19-infected individuals (Lin 2020). The high levels of fear of COVID-19 may also cause irrational and unclear thoughts (Ahorsu et al. 2020). The urgent need for studies on possible prevention strategies was emphasized by Mamun and Griffiths (2020) in order to avoid serious mental health problems such as suicidal thoughts caused by the fear of COVID-19. Therefore, there is a need for assessment tools to uncover the effects of COVID-19 on mental health. In this regard, Ahorsu et al. (2020) developed a valid and reliable instrument to assess the fear of COVID-19. However, there is no available measuring tool to evaluate the fear of COVID19 in Turkey. With an instrument to assess the fear of COVID-19 among Turkish people, it would be possible to uncover the psychological impacts of COVID-19 and to develop psychological interventions to help people cope with the fear being caused by this pandemic. It can also give rise to the scientific studies on the psychological effects of COVID-19. Therefore, this study aims to adapt the Fear of COVID-19 Scale (Ahorsu et al. 2020) into Turkish and investigate the relationships between fear of COVID-19, psychological distress, and life satisfaction.

\section{Method}

\section{Participants and Procedure}

The data were collected through online surveys. By utilizing convenience sampling, we have reached 1304 participants from 75 cities in Turkey. Of all the participants, 917 (70.3\%) were 
females and $387(29.7 \%)$ were males whose ages ranged from 18 to 64 years $(M=29.47$, $\mathrm{SD}=10.54$ ). Detailed information about the participants is presented in Table 1 .

\section{Measures}

In this study, Fear of COVID-19 Scale, Depression Anxiety Stress Scale-Short Form, and Satisfaction with Life Scale were administered.

The Fear of COVID-19 Scale This is a unidimensional 7-item, 5-point Likert scale, developed by Ahorsu et al. (2020). Factor loadings (.66 to .74) and corrected item-total correlation (.47 to .56) of the Fear of COVID-19 Scale were found acceptable. The internal consistency and the test-retest reliability of the scale $(\alpha=.82$ and ICC $=.72)$ was acceptable. The Fear of COVID19 Scale was positively correlated with perceived vulnerability, hospital anxiety, and depression (Ahorsu et al. 2020).

Depression Anxiety and Stress Scale The scale was developed by Lovibond and Lovibond (1995) to provide a self-report measure of anxiety, depression, and stress. The DASS-21 (Depression Anxiety and Stress Scale) was developed from the original DASS-42 by Henry and Crawford (2005) and Mahmoud et al. (2012). The DASS-21 contains three subscales: (1) depression, (2) anxiety, and (3) stress which include 12-items. The DASS-21 was adapted into Turkish by Yilmaz et al. (2017). The factor loadings of the Turkish version of the scale ranged between .41 and .81 and reliability coefficients between .76 and .82 (Yilmaz et al. 2017). In

Table 1 Descriptive information of the participants

\begin{tabular}{lll}
\hline & Frequency & $\%$ \\
\hline Gender & & \\
Female & 917 & 70.3 \\
Male & 387 & 29.7 \\
Education level & & .7 \\
Primary school & 9 & .7 \\
Middle School & 9 & 5.0 \\
High school & 65 & 3.8 \\
Associate degree & 49 & 79.8 \\
Bachelor degree & 1041 & 10.0 \\
Graduate & 131 & 65.0 \\
Marital status & & 35.0 \\
Married & 457 & 31.4 \\
Single & 847 & 68.6 \\
Having a child & & \\
Yes & 409 & 19.6 \\
No & 895 & 79.4 \\
Social isolation & & 1.0 \\
Definitely not leaving home & 255 & 1.7 \\
In compulsory situations & 1036 & 12.9 \\
No problem going out & 13 & 85.4 \\
Covid-19 symptoms so far & & 22 \\
Yes & 168 & \\
Partially & 1114 & \\
No & & \\
\hline
\end{tabular}


this study, the reliability coefficients of the scale were found to be $\alpha=.87, .82$, and .89 , respectively.

Satisfaction with Life Scale It is a unidimensional 5-item, 7-point Likert scale, developed by Diener et al. (1985). The higher scores indicate higher levels of satisfaction with life. The SWLS (Satisfaction with Life Scale) was adapted into Turkish by Durak et al. (2010). The Turkish version of the SWLS was reported to have good validity $(\mathrm{CFI}=.99, \mathrm{IFI}=.99, \mathrm{TLI}=.98, \mathrm{SRMR}=.020$, and $\mathrm{RMSEA}=.043)$ and reliability $(\alpha=.80$; Durak et al. 2010). In this study, the reliability coefficient of the scale was found $\alpha=.83$.

\section{Data Analysis}

Confirmatory factor analysis (CFA) was utilized in order to validate the factor structure of the Fear of COVID-19. As has been previously reported in the literature (e.g., Hu and Bentler 1999; Kline 2015), GFI, NFI, and CFI $\geq .90$ and SRMR $\leq .08$ were considered an indication of acceptable fit. After establishing the factorial structure of the Fear of COVID-19 Scale, the discrimination, difficulty, and informativeness of the scale was examined using the Item Response Theory (IRT; Chalmers 2012), which allows us to have more detailed and reliable information at the individual and item level than classical test theory. These basic aspects of the IRT parameters can be described by the item characteristic curve (ICC), which is a curve shaped like an "S" was used in ICC analysis, and were carried out using the Graded Response Model (GRM) which is appropriate for 5-point Likert-type scales. Based on guidelines proposed by Baker (2001), an $\alpha$ value > 1.0 is considered highly discriminant. Furthermore, corrected item-total correlation coefficients and descriptive statistics for each item were also examined. The correlations between fear of COVID-19, depression, anxiety, stress, and life satisfaction were examined to establish the concurrent validity of the scale. Furthermore, the mediating role of psychological distress on the relationships between fear of COVID-19 and life satisfaction was tested for incremental validity. Process Macro for SPSS developed by Hayes (2018) was adopted to test the proposed mediation model. Five thousand bootstrap replicates were drawn and coefficients for both direct and indirect effects were created in the $95 \%$ confidence interval. Significance was determined when the confidence interval did not include zero. Cronbach's alpha, McDonald's omega, Guttmann's lambda, and composite reliability were examined for the reliability of the Fear of COVID-19 Scale. The analyses of the present study were run using IBM SPSS Statistics 22.0, Amos Graphics 24, Stata 14, and JASP 0.11.1.

\section{Results}

CFA analyses showed that fit indices were all within the acceptable limit $\left[\chi^{2}(13, N=1304)=\right.$ 299.47, $p<.05 ; \mathrm{SRMR}=.061 ; \mathrm{GFI}=.936 ; \mathrm{NFI}=.912$; $\mathrm{IFI}=.915 ; \mathrm{CFI}=.915]$. The factor loadings of the Fear of COVID-19 Scale were found significant ranging from .484 to .723. Item analysis results for Fear of COVID-19 Scale is given in Table 2.

Results of the IRT are shown in Fig. 1 and Table 3. 
Table 2 Statistics on the items of the Fear of COVID-19 Scale

\begin{tabular}{llllll}
\hline & Factor loading & Corrected item-total correlation & $M(\mathrm{SD})$ & Skewness & Kurtosis \\
\hline Item 1 & .697 & .608 & $3.46(1.04)$ & -.503 & -.466 \\
Item 2 & .709 & .616 & $3.63(1.10)$ & -.802 & -.202 \\
Item 3 & .484 & .506 & $1.79(.88)$ & 1.328 & 1.846 \\
Item 4 & .708 & .632 & $3.07(1.25)$ & -.185 & -1.124 \\
Item 5 & .723 & .635 & $3.67(1.08)$ & -.829 & -.047 \\
Item 6 & .623 & .625 & $2.01(1.01)$ & 1.003 & .404 \\
Item 7 & .647 & .624 & $2.40(1.15)$ & .495 & -.822 \\
\hline
\end{tabular}

As displayed in Table 3, all $\alpha$ values were higher than 1.0. Hence, IRT results revealed that the Fear of COVID-19 Scale had appropriate item difficulty and the ability to discriminate between performers and non-performers.

Following IRT analyses, concurrent validity was evaluated by computing Pearson's correlations of the Fear of COVID-19 Scale with the depression, anxiety, stress, and life satisfaction as validity scales. Data analysis revealed significant positive correlations between the Fear of COVID-19 Scale and depression $(r=.38, p<.001)$, anxiety $(r=.55, p<.001)$, and stress $(r=.47, p<.001)$. On the other hand, the Fear of COVID-19 Scale is significantly and negatively correlated with life satisfaction, $r=-.20, p<.001$.

The incremental validity of the Fear of COVID-19 Scale was also tested in a process model linking fear of COVID-19 to life satisfaction via depression, anxiety, and stress. Findings for the regression pathways and the direct and indirect effects examined for mediation are presented in Fig. 2 and Table 4.

Figure 2 shows the role of fear of COVID-19 on life satisfaction through depression, anxiety, and stress. When the direct effects were examined (see Table 4 ), fear of the COVID-19 significantly positively predicted depression $(B=.292, t=18.84, p<.001)$, anxiety $(B=.321, t=23.94$, $p<.001)$, and depersonalization $(B=.396, t=19.11, p<.001)$. Moreover, bootstrap analysis confirmed the indirect effects of the fear of COVID-19 and life satisfaction, with 95\%CI and did not contain zero. Therefore, depression (bootstrap value $=-.143,95 \% \mathrm{CI}=-.177,-.112$ ), anxiety (bootstrap value $=-.078,95 \% \mathrm{CI}=-.124,-.033)$, and stress (bootstrap value $=-.126,95 \% \mathrm{CI}=$ $-.162,-.091)$ mediated the relationship of fear of COVID-19 on life satisfaction.

Finally, Cronbach's alpha, McDonald's omega, Guttmann's lambda, and composite reliability were assessed for the reliability of the Fear of COVID-19 Scale. The results showed that the Cronbach's alpha $(\alpha=.847)$, McDonald's omega $(\omega=.849)$, Guttmann's lambda $(\lambda 6=.844)$, and composite reliability $(\mathrm{CR}=.842)$ were found to be satisfactory.

\section{Discussion}

At the time of writing, the coronavirus pandemic is undoubtedly the most important problem all around the world. Apart from its impacts on physical health, COVID-19 also has significant effects on mental health. Hence, there is a need for assessment tools to investigate the effects of COVID-19 on people's mental health. The aim of this study was to adapt The Fear of COVID19 Scale (Ahorsu et al. 2020) into Turkish and investigate the relationships between fear of COVID-19, psychological distress, and life satisfaction. The CFA confirmed the unidimensionality of the 7-item scale on a Turkish sample. All factor loadings of the Fear of COVID-19 Scale were also found statistically significant. Therefore, we can conclude that the Turkish 

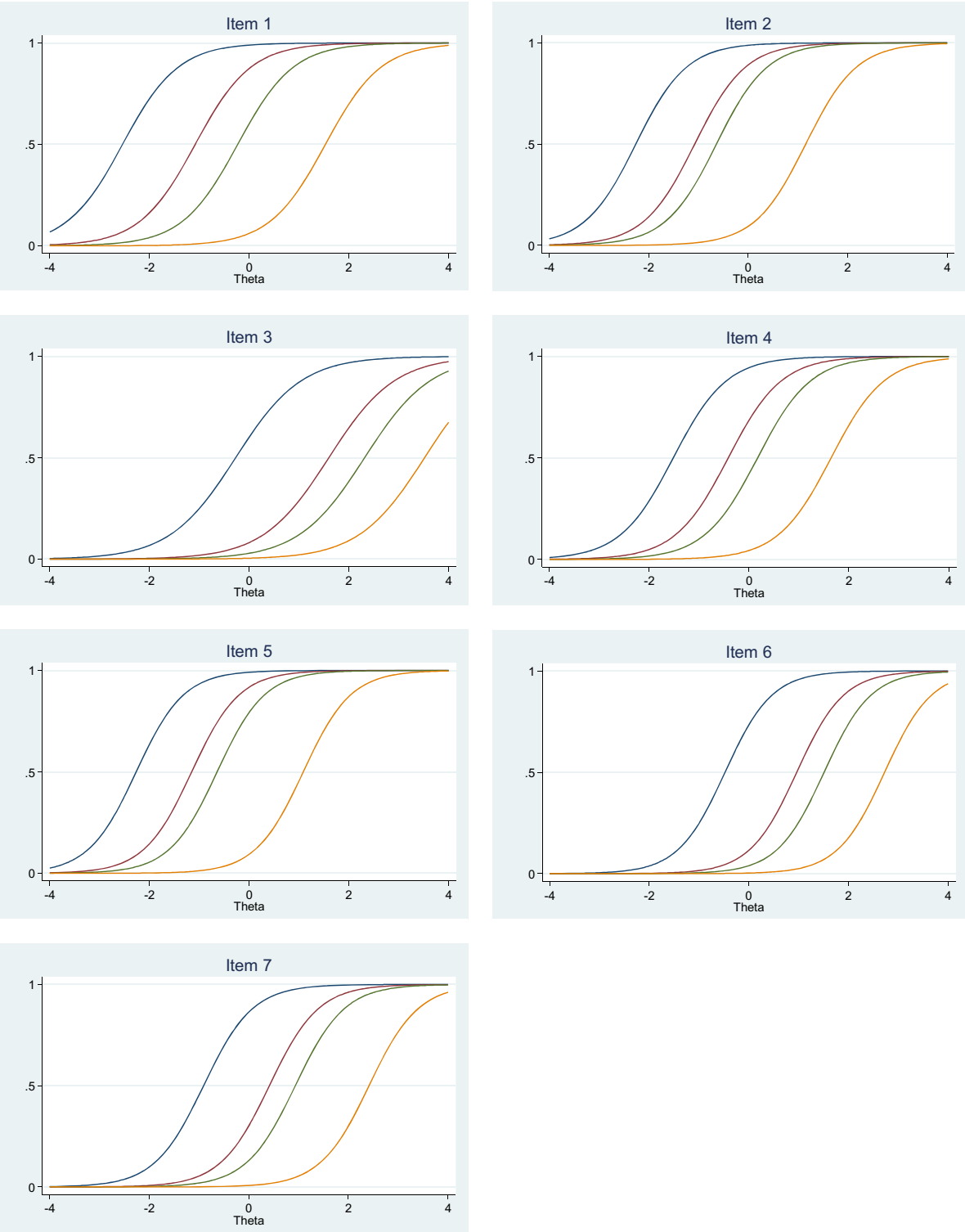

Fig. 1 Item characteristics curve for the Fear of COVID-19 Scale

version of the Fear of COVID-19 Scale supported the structure of the original scale by Ahorsu et al. (2020).

In this study, Item Response Theory (IRT) was used to assess the validity of items of the Fear of COVID-19 Scale. IRT and classical test theory (CTT) are two different measurement frameworks. CTT has a significant shortcoming. The summed scores are sequential; statistical inferences derived from sequence scores are likely to be invalid since it is assumed that raw scores should be regarded as a range scale (Jafari et al. 2012). To illustrate, CTT uses a 5-point Likert scale (strongly disagree, disagree, neither agree nor disagree, agree, and strongly agree) 
Table 3 Item Response Theory estimates for the Fear of COVID-19

\begin{tabular}{|c|c|c|c|c|c|}
\hline \multirow[b]{2}{*}{ Item } & \multicolumn{5}{|c|}{ Item parameter estimates } \\
\hline & $\alpha$ & $b_{1}$ & $b_{2}$ & $b_{3}$ & $b_{4}$ \\
\hline 1 & 1.78 & -2.54 & -1.08 & -.22 & 1.53 \\
\hline 2 & 1.95 & -2.26 & -1.07 & -.63 & 1.15 \\
\hline 3 & 1.51 & -.27 & 1.59 & 2.31 & 3.52 \\
\hline 4 & 1.87 & -1.52 & -.42 & .16 & 1.64 \\
\hline 5 & 2.09 & -2.27 & -1.15 & -.64 & 1.08 \\
\hline 6 & 2.12 & -.47 & .96 & 1.51 & 2.72 \\
\hline 7 & 2.03 & -.91 & .41 & .93 & 2.41 \\
\hline
\end{tabular}

as an interval scale in which items were scored from zero to four where the differences between the five points may not be equal whereas the IRT employs logistic regression which allows us to predict the underlying capabilities of participants and item challenges. Hence, the interval-level measurement of each participant's COVID-19 fear and the interval-level measurements of item challenges were examined. The characteristics and validity of the items are examined using IRT, and the fitness of the items was calculated. Based on IRT results, the item challenges and characteristics of the Fear of COVID-19 Scale were appropriate.

The concurrent validity analysis revealed significant positive correlations between the fear of COVID-19, depression, anxiety, and stress. The fear of COVID-19 was found negatively correlated with life satisfaction. Additionally, the fear of COVID-19 was shown to increase depression, anxiety, and stress and to decrease life satisfaction. Limited number of studies also demonstrated that the fear of COVID-19 led to higher levels of psychological distress and poor mental health. For instance, Ahorsu et al. (2020) suggested that the fear of COVID-19 has positive associations with hospital anxiety and depression. Constant exposure to the news about worldwide fatalities or infection rate of the pandemic has led individuals to experience fear, anxiety, and depression. The worries about the risk of getting infected increase fear among the general public (Lin 2020).

a

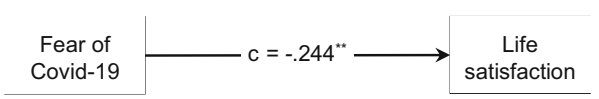

C

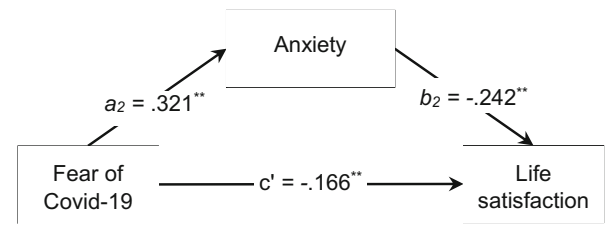

b

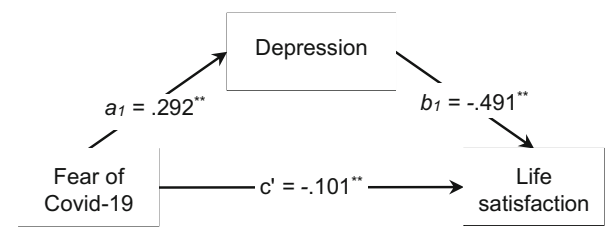

d

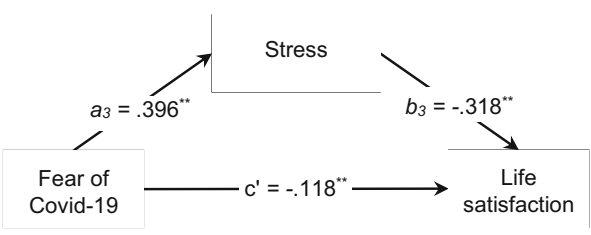

Fig. 2 Mediated outcomes on life satisfaction (panels B, C, and D) showing indirect effects of fear of COVID-19 through psychological distress 
Table 4 Fear of COVID-19 predicts life satisfaction through psychological distress

\begin{tabular}{lllll}
\hline Variable & $B$ & SE & $t$ & $p$ \\
Direct effect & & & & \\
Fear of COVID-19 $\rightarrow$ depression & .292 & .019 & 14.84 & .001 \\
Fear of COVID-19 $\rightarrow$ anxiety & .321 & .013 & 23.94 & .001 \\
Fear of COVID-19 $\rightarrow$ stress & .396 & .021 & 19.11 & .001 \\
Fear of COVID-19 $\rightarrow$ life satisfaction ${ }^{\mathrm{a}}$ & -.244 & .033 & -7.388 & .001 \\
Variable & Value & SE & LL 95\% CI & UL 95\% CI \\
Bootstrap results for indirect effect & & & & -.112 \\
Fear of COVID-19 $\rightarrow$ depression $\rightarrow$ SWL & -.143 & .016 & -.177 & -.033 \\
Fear of COVID-19 $\rightarrow$ anxiety $\rightarrow$ SWL & -.078 & .023 & -.124 & -.091 \\
Fear of COVID-19 $\rightarrow$ stress $\rightarrow$ SWL & -.126 & .018 & -.162 & \\
\hline
\end{tabular}

Bootstrap sample size $=5000 ; L L$, lower limit; $U L$, upper limit; $C I$, confidence interval; $S W L$, life satisfaction a Total effect

Finally, multiple techniques were used to establish the reliability of the Fear of COVID-19 Scale. Cronbach's alpha for the original scale was .82 (Ahorsu et al. 2020). In the present study, Cronbach's alpha, McDonald's omega, Guttmann's lambda, and composite reliability were assessed and reliability coefficients for total scale was found .84 . The findings also indicated that the scale had a reliability coefficient over .70 which met the criterion of acceptable reliability recommended by Nunnally (1978). Thus, we can conclude that Turkish Fear of COVID-19 Scale is a reliable measurement tool.

The present study has some limitations. First, the data were collected using a non-clinical sample. Therefore, results may not be generalizable to a clinical sample. Second, the findings of this study were based on self-report data which has the risk of source bias. Third, although more than one reliability analyses were carried out, the test-retest reliability of the scale cannot be examined for the sake of the scale to be used in Turkish language promptly. Therefore, we do not have any information regarding the temporal reliability of the scale. Despite its limitations, the findings of this study will help mental health professionals to deal with the mental health problems of general public generated by COVID-19. Additionally, the adaptation study of The Fear of COVID-19 Scale was conducted using a large sample of participants with varying ages from 75 cities in Turkey. The use of the Fear of COVID-19 Scale can give a rise to the assessment of the psychological impacts of COVID-19 on individuals. Mental health professionals should investigate the relationships between the fear of COVID-19 and panic attack, obsessive compulsive disorder, and stigma. Finally, Pakpour and Griffiths (2020) emphasized the importance of determining the risk groups based on socio-demographic variables in order to develop prevention programs to help overcome fear of COVID-19. Therefore, psychological interventions can be designed to alleviate the traumatic effects of COVID-19 by using the Fear of COVID-19 Scale.

\section{Compliance with Ethical Standards}

All procedures performed in studies involving human participants were in accordance with the ethical standards and with the Helsinki Declaration and its later amendments or comparable ethical standards.

Conflict of Interest The authors declare that they have no conflict of interest.

Informed Consent Informed consent was obtained from all individual participants included in the study. We did not receive any financial support for the research, authorship, and/or publication of this article. 


\section{References}

Ahorsu, D. K., Lin, C. Y., Imani, V., Saffari, M., Griffiths, M. D., \& Pakpour, A. H. (2020) The Fear of COVID19 Scale: development and initial validation. International Journal of Mental Health and Addiction, 1. https://doi.org/10.1007/s11469-020-00270-8

Baker, F. B. (2001). The basics of item response theory (2nd. Ed.). Retrieved from https://eric.ed.gov/?id= ED458219.

Chalmers, R. P. (2012). mirt: a multidimensional item response theory package for the R environment. Journal of Statistical Software, 48(6), 1-29.

Diener, E. D., Emmons, R. A., Larsen, R. J., \& Griffin, S. (1985). The satisfaction with life scale. Journal of Personality Assessment, 49(1), 71-75.

Durak, M., Senol-Durak, E., \& Gencoz, T. (2010). Psychometric properties of the satisfaction with life scale among Turkish university students, correctional officers, and elderly adults. Social Indicators Research, 99(3), 413-429.

Hayes, A. F. (2018). Introduction to mediation, moderation, and conditional process analysis: a regressionbased approach. New York: Guilford Publications.

Henry, J. D., \& Crawford, J. R. (2005). The short- form version of the Depression Anxiety Stress Scales (DASS21): construct validity and normative data in a large non- clinical sample. British Journal of Clinical Psychology, 44(2), 227-239.

Hu, L. T., \& Bentler, P. M. (1999). Cutoff criteria for fit indexes in covariance structure analysis: conventional criteria versus new alternatives. Structural Equation Modeling: A Multidisciplinary Journal, 6(1), 1-55.

Jafari, P., Bagheri, Z., Ayatollahi, S. M. T., \& Soltani, Z. (2012). Using Rasch rating scale model to reassess the psychometric properties of the Persian version of the PedsQL TM 4.0 Generic Core Scales in school children. Health and Quality of Life Outcomes, 10(1), 27.

Kline, R. B. (2015). Principles and practice of structural equation modeling (4th ed.). New York: Guilford Publications.

Lin, C.-Y. (2020). Social reaction toward the 2019 novel coronavirus (COVID-19). Social Health and Behavior, 3(1), 1-2. https://doi.org/10.4103/SHB.SHB_11_20.

Lovibond, P. F., \& Lovibond, S. H. (1995). The structure of negative emotional states: comparison of the Depression Anxiety Stress Scales (DASS) with the Beck Depression and Anxiety Inventories. Behaviour Research and Therapy, 33(3), 335-343.

Mahmoud, J. S. R., Staten, R. T., Hall, L. A., \& Lennie, T. A. (2012). The relationship among young adult college students' depression, anxiety, stress, demographics, life satisfaction, and coping styles. Issues in Mental Health Nursing, 33(3), 149-156.

Mamun, M. A., \& Griffiths, M. D. (2020). First COVID-19 suicide case in Bangladesh due to fear of COVID-19 and xenophobia: possible suicide prevention strategies. Asian Journal of Psychiatry, 51(102073), 102073. https://doi.org/10.1016/j.ajp.2020.102073.

Nunnally, J. C. (1978). Psychometric theory. McGraw-Hill Book Company.

Pakpour, A. \& Griffiths, M. D. (2020). The fear of COVID-19 and its role in preventive behaviors. Journal of Concurrent Disorders. Advance online publication. https://concurrentdisorders.ca/2020/04/03/the-fear-ofcovid-19-and-its-role-in-preventive-behaviors/

Schimmenti, A., Billieux, J., \& Starcevic, V. (2020). The four horsemen of fear: an integrated model of understanding fear experiences during the COVID-19 pandemic. Clinical Neuropsychiatry, 17(2), 41-45.

Wang, C., Pan, R., Wan, X., Tan, Y., Xu, L., Ho, C. S., \& Ho, R. C. (2020). Immediate psychological responses and associated factors during the initial stage of the 2019 coronavirus disease (COVID-19) epidemic among the general population in China. International Journal of Environmental Research and Public Health, 17(5), 1729.

Xiang, Y.-T. (2020). Timely mental health care for the 2019 novel coronavirus outbreak is urgently needed. Lancet Psychiatry, 7, 228-229.

Yilmaz, O., Boz, H., \& Arslan, A. (2017). The validity and reliability of depression stress and anxiety scale (DASS 21) Turkish short form. Research of Financial Economic and Social Studies, 78-91.

Zhang, J., Wu, W., Zhao, X., \& Zhang, W. (2020). Recommended psychological crisis intervention response to the 2019 novel coronavirus pneumonia outbreak in China: a model of West China Hospital. Precision Clinical Medicine, 3(1), 3-8.

Publisher's Note Springer Nature remains neutral with regard to jurisdictional claims in published maps and institutional affiliations. 